\title{
Revealable Volume Displays: 3D Exploration of Mixed-Reality Public Exhibitions
}

\author{
Fatma Ben Guefrech* \\ Florent Berthaut \\ Patricia Plénacoste \\ CRISTAL, Université de Lille, France
}

Yvan Peter ${ }^{\S}$

Laurent Grisoni ${ }^{\text {II }}$

\begin{abstract}
In this paper, we present a class of mixed-reality displays which allow for the 3D exploration of content in public exhibitions. The shared experience of the exhibition and the preservation of artworks are two very important aspects of these contexts, in particular for museum exhibits. The use of display cases as a protection tool is substantially accepted. It decreases the risks of damages to artworks and cultural materials hosted in museums. In addition, the transparent panels create a reflection of the visitors inside the display case. This reflection can be used to augment and interact in 3D with the exhibited content, by coupling Spatial Augmented-Reality and Optical Combiners. We call such a combination a Revealable Volume Display (RVD). It allows visitors to reveal information placed freely inside or around protected artefacts, visible by all, using their reflection in the panel. However, it may also suffer from unfamiliar gestures and disrupted depth perception cues, making 3D exploration of content difficult. In this paper, we first discuss the implementation of RVDs, providing both projector-based and mobile versions. We then present a design space that describes the interaction possibilities that they offer. Drawing on insights from a field study during a first exhibition, we finally propose and evaluate techniques for facilitating 3D exploration with RVDs.
\end{abstract}

Index Terms: Human-centered computing-Human computer interaction (HCI) - Interaction devices-Graphics input devices Human-centered computing-Human computer interaction (HCI) - Interaction devices-Displays and imagers Human-centered computing-Human computer interaction (HCI)-Interaction paradigms-Mixed / augmented reality

\section{INTRODUCTION}

Mixed-reality has known in the past years impressive technological progress which democratised its use in our daily lives. It is now common in several aspects of human activity (games, distant social interaction, virtual tourism, ...). However, current approaches are still mostly limited to situations that are compatible with quite fragile and expensive (even though more and more accessible) devices. Consequently, when envisaging public situations, unless users come with their personal equipment, it is still difficult in practice to reasonably imagine to actually use such mixed-reality systems. Such interaction scenarios, where visitors are focused on their personal devices and interactions, also strongly limit a collectively shared visit experience.

In public situations where mixed-reality is aimed to combine digital information with existing (tangible) artefacts, one will also wish

\footnotetext{
*fatma.benguefrech@univ-lille.fr

†florent.berthaut@univ-lille.fr

†atricia.plenacoste@univ-lille.fr

\$yvan.peter@univ-lille.fr

Ilaurent.grisoni@univ-lille.fr
}

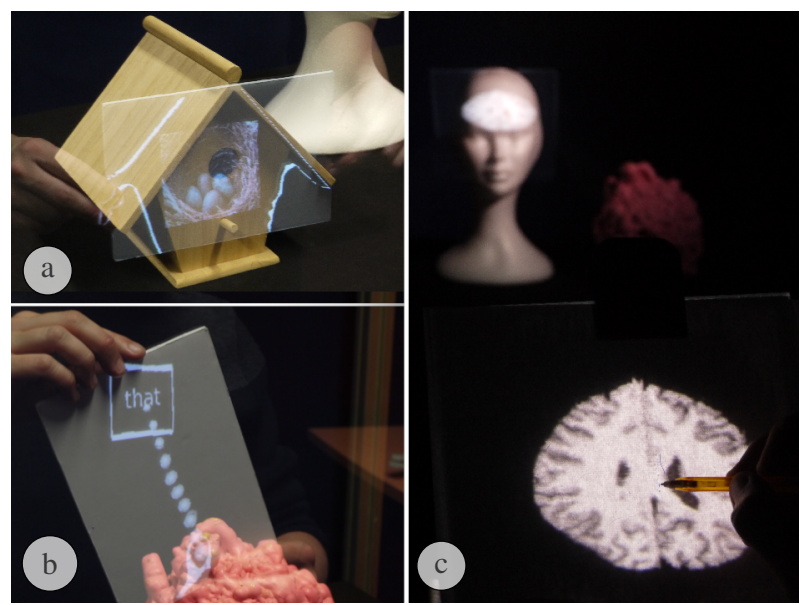

Figure 1: Revealable Volume Displays enable various content and interactions with mixed-reality exhibitions : (a) Image layers inside a physical object with highlighted contours, (b) Text annotations attached with 3D guides (d) Exploring and annotating a revealed MRI scan inside a head using a transparent panel and paper

to protect the artefacts from the visitors (for various reasons, including protection from stealing, or degradation). Indeed, some specific public exhibitions (such as museums) have even more constraints on the preservation of artefacts, such as preserving sensitive artefacts from temperature, humidity and pollutants and the risks of accidental damage. Glass display cases are a common mean to present fragile, rare or valuable objects coming from cultural or artistic heritage in a safe way. In addition, well-lit display cases allow for showing each artefact in its full potential. The challenge faced by mediators is then to find a way to transform the closed glass display cases into smart and attractive exhibitions that can catch the visitors' attention. Looking at these multiple constraints, it appears that public exhibitions would benefit from novel mixed-reality technologies which would engage the visitors while preserving the physical and social aspects of the exhibition ecosystem.

In this paper, we present a class of Mixed-Reality displays that we call Revealable Volume Displays (RVDs), which are particularly suited for this context of public exhibitions. They rely on users actions to reveal 3D virtual content inside or around exhibited physical objects, as shown on Figure 1, using the reflection in the glass panel which protects them. RVDs ensure that the visitor's attention is focused on the actual exhibits, avoiding the "heads-down" effect of mobile mixed-reality; they entirely rely on the user's interaction to display content, maximising the engagement with the exhibition; they provide shared augmentations, unlike mixed-reality headsets, correctly perceived by all visitors, hence opening social interactions on projected digital information; they leave physical artefacts safely protected and unaltered by the display. 
Our contribution is four-fold :

- We describe the concept and implementation of RVDs

- We propose a design space that shows the augmentation and interaction possibilities that they offer

- We investigate visitors' responses to RVDs with an in-situ experiment on a first prototype

- We evaluate techniques and propose design guidelines to facilitate the use of such displays

\section{Related Work}

In the following section, we present related work on the use of mixed-reality in public exhibitions, especially in museums. We then describe other augmented and mixed reality display technologies that focus on public / shared virtual content. Finally we focus on studies that aim at guiding the exploration of digital content, in various contexts.

\subsection{Mixed-Reality for Mediation}

In the context of mediation (museums, products exhibition, ...), Augmented (AR) and Mixed-Reality (MR) technologies offer the opportunity to combine digital information with exhibited physical objects. We can cite as an example mobile AR applications for museums which offers visitors a device to explore exhibits. For example, Augmented Reality Stories at Acropolis Museum presented by Keil et al. [22] shows how mobile devices bring augmented colours and stories to art pieces at the Acropolis Museum through AR developed by the CHESS project (Cultural Heritage Experiences through Sociopersonal interactions and Storytelling). These applications exploit a smartphone back camera to integrate virtual objects on the physical space or to display data providing useful information for visitors . In these cases, AR technology might make finding information in the museum easier and enrich the visitors' experience.

Bekele and al. recently realised a survey of Augmented, Virtual and Mixed Reality for Cultural Heritage (CH) [2]. They classified projects according to the technologies used in $\mathrm{CH}$ projects for tracking and registration, virtual environment modelling, devices and interaction interfaces as well as the purpose of the project : education, exhibition enhancement, exploration, reconstruction, and virtual museums. The former categories provides the design dimensions of an AR application. Ibáñez \& Delgado-Kloos have realised a review of Augmented Reality for STEM learning on the period 2010 - 2017 [19]. Their study is not restricted to the use of AR in cultural heritage sites and museums but it shows that the AR applications tend to use more than one type of media to convey information. Text and 2D images are the most used media but animations, 3D objects and videos are also quite common. The type of activities can be classified in three categories : exploration, simulation and game. In this paper, we focus mostly on the exploration aspect, with displays dedicated to this activity.

Liarokapis et al. [23] state that AR exhibition success is highly related to the level of realism achieved. Especially, with AR for museum exhibition, visitors have high expectations that the visualised information will be naturally presented in an entertaining manner. According to Boisvert et al. [7] two necessary steps are required to ensure visitors engagement in museums: the attractiveness of the interface and the holding power. Attraction is defined as the measurement of visitors who stop at the exhibit while the holding power is time spent by visitors in the exhibit. The study by Yahya [36] indicates that attraction and holding power are important variables for understanding the learning environment of the museum. In addition, holding power affects the visitor participation during visiting the exhibition. These information are important indicators of museums' ability to engage visitors' attention and maintain their interest. Even though AR and MR technologies can motivate users to interact in new ways with exhibits, according to Hsi [18], they may suffer from a "heads-down" effect where the interaction with the device takes most of the attention of the visitor, hindering the experience as well as the interaction with other visitors $[13,18,26]$. Another dimension of the engagement of museum visitors is collaboration and social experience. As stated by vom Lehn et al., social interaction and collaboration are becoming increasingly important to the design and development of exhibitions [34]. Indeed, discussion between visitors fosters exchanging point of views and elaborating the reasons for their opinion on the exhibits which leads to learning and a more thorough experience [17].

In this paper, we present a new class of mixed-reality displays that take these constraints into account, i.e. where user engagement is ensured by relying on their interaction for the display of virtual content and where the augmentations are displayed directly on the physical objects, visible by all visitors.

\subsection{Mixed-reality displays for public contexts}

In this section we consider MR displays that enable multiple users to collectively perceive virtual content augmenting physical objects. Kajita et al. propose SkyAnchor a glass-free mixed-reality solution that overlaps virtual content with a physical object [20]. Their solution enables a zero-latency imaging while the object moves by attaching the light source to the object. The tracking is based on the surface on which the object is (capacitive markers), so one can not lift it. The size of the image is also dependant on the size of the system. Karnik et al. describe Mustard [21], a multiviewer see-through display to provide information about objects in a glass cabinet. The system is based on two liquid crystals panels between which the objects are placed. The front panel is used to display information while the back panel serves as a hole-mask that restricts what a user can view at one position. This enables the systems to have location specific views for different users. The user does not have the means to interact with the system to change the information displayed. Ridel et al. propose another way to explore information by the controlled projection of 3D visualisation on top of previously scanned artefacts [28]. The user can drive the projection using a revealing flashlight. This interactive device provides three characteristics : spot (what to show), distance (size and illumination) and angle (intensity of the augmentation). Projected information is however limited to the surface of the exhibited objects and might damage fragile artefacts. The same authors also describe EgoSAR, an augmented reality display that superimposes personalised virtual content for different viewers around a physical object [27]. The system is based on retro-reflective material combined with multiple light sources. The different views are however associated to specific locations unless the user is equipped with a portable projector in an alternative setting. Martinez et al. explore an idea similar to what we present in this paper [25]. They use it as an example of how optical combiners can be used to augment physical spaces. However, they do not evaluate the interaction with the system or provide guidelines on the design of augmented museum cabinets using such a system.

\subsection{Guiding exploration of virtual content}

Exploration of virtual content in public exhibitions can be made difficult by the amount of accessible information and the type of display technology used, and can therefore benefit from guiding. Presenting 3D visualisations in public spaces is in fact identified as an important challenge by Besançon et al. [5]. Ynnerman et al. [1] propose the term exploranation as the merging of exploratory and explanatory visualisation. It offers new possibility for mediation in public settings, through adaptive interfaces, sensitive guidance to enhance the experience of exploration.

Another technique is to have other users demonstrate gestures which need to be performed, in order to generate a shared knowledge. The study by Cash et al. [10] involves the gestures repetition as a tool to build shared understanding. Yasui et al. [37] demonstrate 
that gesture repetition helps building a shared knowledge within participants.

Another possibility is to provide visual guides. In the context of 2D interfaces, Burigat et al. [8] propose a technique to display large information spaces on the limited screens of mobile devices by indicating the direction of Points of Interest (PoI) outside the displayed part of a map. In the context of 3D Virtual Environments, according to Chen et al. [14], including navigation aids to guide the exploration process provides a significant learning effect. Prior studies [16] also confirm that guiding the user in a Virtual Reality Museum experience with continuous inputs in space and time stimulates an improved experience.

In the context of Mobile Augmented Reality, Schinke et al. [30] propose a technique that embeds arrows pointing at nearby PoI. Their study confirms that 3D arrows help users estimate the position of objects more precisely than a mini-map. In addition, according to Carmo et al. [9], the integration of PoI allows for a better knowledge of the environment and a better user guidance through a collection of clues on off-screen objects and a 2D map with a radar view. Recently, the study of Galatis et al. [15] suggests KnossosAR as an outdoor AR tool for the visitors of cultural heritage sites. This mobile application deals with the occlusion problem and how to handle occluded PoI Their user evaluation trials confirms that guides stimulate the interest of participants to physically explore the archaeological site. In the context of Spatial Augmented-Reality, Sodhi et al. [32] explore how on-body projected visual hints could be used for movement guidance. Schmidt and al. [31] explore a spatial augmented reality environment through a floor projected guidance user interface. Their aim is to support the storytelling process by guiding users to regions of interest and ideal viewpoints. In this paper, we investigate similar techniques to guide users through the exploration of RVDs.

\section{Revealable Volume Displays : Implementation AND DESIGN SPACE}

In this section, we first describe the principle of Revealable Volume Displays, their implementation and the content and techniques that they enable.

\subsection{General approach}

RVDs aim at satisfying a number of constraints for mixed-reality public exhibitions, which are not all taken into account by existing MR displays:

1. They preserve the exhibited objects by leaving them behind protective panels and not projecting directly on them. This configuration also protects the exhibited artefacts from the visitors' hands

2. They keep visitors focused on the physical objects, not on an external screen

3. They encourage the exploration [1] of the museum exhibits as the virtual content is only visible when visitors are interacting with it, unlike with AR headsets

4. They allow all visitors, i.e. not only the ones interacting as it would be the case with an AR headset, to perceive the augmentations consistently, which encourages personal trajectories and collaborations [3].

As shown in Figure 2.a, RVDs utilise the glass cabinet that protects exhibited objects. When a physical surface, such as a hand, body part or handheld object is placed and moved in front of the cabinet, its reflection is perceived as moving through the volume inside the cabinet, intersecting the exhibited objects. We use this principle to reveal virtual content placed inside the cabinet by re-projecting the intersections between the reflected surface and the virtual content onto the surface. For example, in Figure 2.a the reflection of a white panel is being moved through a naturalised bird and the panel is sensed by a depth camera. Overlapping the bird, a virtual box was

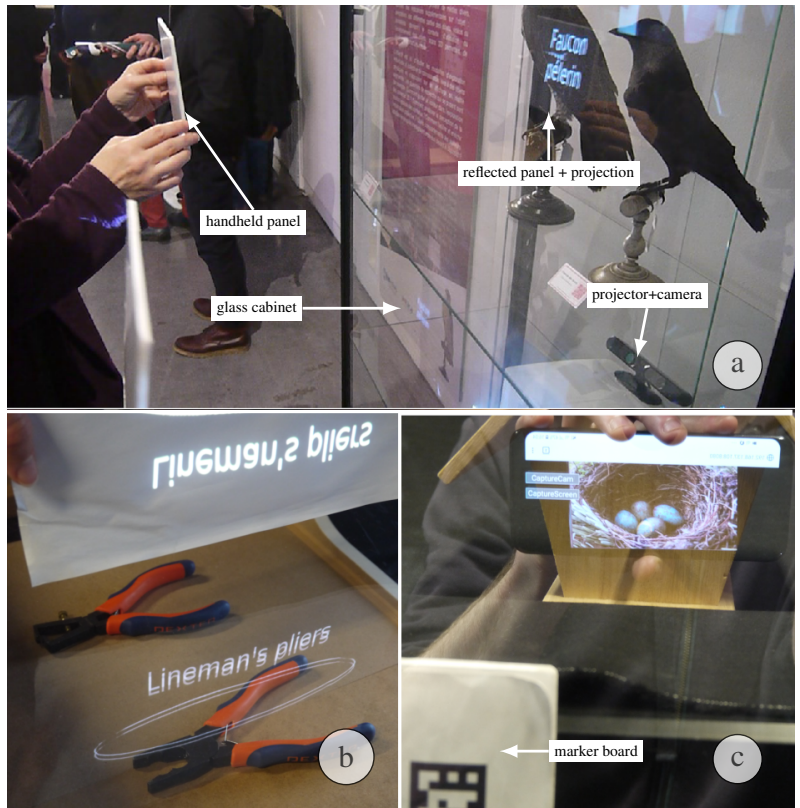

Figure 2: Implementation of Revealable Volume Display : (a) Projector + depth camera implementation with a visitor revealing the name of an exhibited bird, (b) Horizontal configuration with objects below the glass panel and text annotations revealed using a sheet of paper, (c) Mobile implementation of RVD

placed which contains a texture with the name of the bird. When the reflection of the panel intersects the box, this intersection, or slice, is projected back onto the panel by the projector, and is perceived as inside the bird. Existing glass panels can be used, provided they are flat (e.g. without bumps). Thicker panels will however create double reflections that might disturb the perception of for example. Lighting must also sometimes be adapted. If the inside or background of the cabinet are too bright, they might hide the reflected content.

RVDs draw inspiration from Slicing Displays $[4,12]$, AR displays which use Optical Combiners [25], swept-volume displays [33] and Spatial Augmented reality [6]. Like swept-volume displays, RVDs display voxels, i.e. elements composing virtual content placed in 3D in the physical space. In doing so, they provide depth cues such as stereopsis without requiring head-tracking or a stereoscopic technology. Unlike swept-volume displays, but like slicing displays, the entire display of information relies on visitors actively exploring the content. If the content is not actively revealed by a user, it is not visible. Unlike swept-volume displays and slicing displays, RVDs also allow for augmenting physical objects with virtual content placed inside or around them. Like Spatial AR displays, the content displayed is visible by all users in the physical space, although only when looking through the same panel, but RVDs also allow for placing content in mid-air, i.e. not necessarily on a pre-existing projection surface in the target space. Finally, like Optical Combiners based displays, due to the flatness of the optical combiner (here the glass cabinet) as explained by Martinez and al. [25], the virtual content is perceived consistently (at the same position) by all visitors independently from their point of view. Unlike many other Optical Combiners based displays however, such as "Pepper's Ghost"-type displays, RVDs allow for displaying virtual content placed anywhere in a volume, not on a fixed surface.

\subsection{Implementation}

RVDs allow one to place 3D meshes (primitive shapes, arbitrary 3D models) at any position within the volume inside the glass cabinet. 
These meshes will hold the different types of content (solid or gradient colours, text, 2D and 3D textures, video textures). The tracking and display can be implemented in two ways. Our most common implementation relies on a depth camera and a projector. In this case, the software part of our system resembles the augmented reality pipeline described by Berthaut et al. [4], with additional filtering possibilities described below. The slicing process is entirely done on the GPU in three rendering passes. It requires that the projector and camera are calibrated to retrieve the projection and view matrices that will be used to render the 3D scene of virtual content. A 3D mesh is created from the depth image captured by the camera and rendered to a first depth texture from the projector point of view. The second pass selects all pixels of the back faces of the virtual objects which are behind pixels of the depth texture and renders them to a second texture. In the third pass, pixels of the front faces are finally selected for rendering if they are located before pixels in the depth texture and if they match pixels of the same object in the second texture. Their colour is then computed based on the type of content contained in their corresponding 3D mesh and on the 3D coordinates of the depth texture pixel relative to the mesh.

Because our system aims at displaying text annotations and images, we added a filtering technique that reduces both temporal and spatial noise due to the low resolution (640x480 at 30fps or 320x240 at 60fps) of the depth camera (Asus Xtion or a similar device based on projected IR pattern). To that extent, we combine a temporal filter using a $1 €$ filter [11] that removes the small jitter from the depth image while preserving fast movements of the physical surface, and a spatial filter that averages the depth positions to flatten captured physical surfaces. Both filters are computed on the CPU and passed as an alternative depth image to the slicing pipeline. The first pass then selects each pixel between the last depth image from the camera and the filtered depth image depending on a difference threshold. In practice, this leads to very stable depth meshes when the physical surfaces are relatively motionless and accurate displacement when they are moving. Compared to other approaches to real-time depth image filtering such as using the albedo [35], our approach only relies on the depth image and is therefore less dependent on the lighting conditions of exhibitions.

In addition to this projector / depth camera combination, a second simpler and preliminary prototype displayed in Figure 2.c shows how RVDs can be implemented using a mobile device. Here a web page can be served to the mobile device of visitors containing an application written in Javascript and WebGL. It uses the Aruco [29] marker-based tracking library to obtain the position of the virtual camera relatively to a marker board placed inside the museum cabinet. This camera is configured as an orthographic camera with a very close near plane, effectively resulting in only a slice of the virtual content being shown on the screen.

\subsection{Design space}

In this section, we propose a set of dimensions which describe the design opportunities offered by a Revealable Volume Display.

\subsubsection{Role of virtual content}

RVDs allow virtual content to play various roles with respect to augmented physical objects. Although these are similar to roles available with other types of Augmented Reality Displays, RVDs present the content in a unique way : perceptually aligned with physical objects for all the users in front of the display, but only as a fraction of the entire virtual content. This content can therefore be used with one or a combination of the following roles :

- to reveal the inside of the physical object, e.g a slice of layers inside the bird house show on Figure 1.a.

- to annotate parts of a physical object to explain or direct the user to other information, as shown on Figure 1.b.
- to extend a physical object with virtual content, e.g. completing a partial archaeological artefact with a virtual reconstruction of the remainder.

- to highlight the contour / surface of the physical object, as shown on Figure 1.a on the contours of the bird house.

- to complement the object with additional content, e.g. a video which presents the usage context of an artefact.

\subsubsection{Use of depth in the virtual content}

In RVDs, depth is a very specific dimension since the XY plane acts as a $2 \mathrm{D}$ screen representing a slice of content visible by all. Depth can therefore have the following uses :

- Spatial when one moves through layers in a 3D texture, such as shown on Figure 1.c.

- Temporal when one moves through positions (e.g. frames) in a video or animation such as seen on Figure 1.a.

- Semantic when one moves through semantically organised content (e.g. the chronology of evolution of an object across history)

\subsubsection{Exploration guidance}

Because RVDs heavily rely on exploration of virtual content, how the user is guided through this exploration is an important part of the design. This exploration can be :

- Spontaneous when user moves freely to explore the virtual scene

- Guided when the exploration is triggered and guided by e.g. annotations or sounds which allow for proposing a trajectory

\subsubsection{Implementation dependent interactions}

Depending on the implementation chosen for the RVD, various interaction possibilities arise. The first implementation uses the projector + depth camera system, with the user holding a unprepared opaque panel or using their body or clothes to reveal the content. The second implementation also uses projector + depth camera together with prepared opaque panels featuring printed tags, such as shown in Figure 4.a. The third implementation also relies on a projector + depth camera setup combined with a transparent (e.g. acrylic) panel to which is attached a piece of paper, on which one can write. The revealed content is then visible by transparency on the other side of the panel and can be traced or annotated. This version is shown in Figure 1.c. Finally the fourth implementation is the mobile display shown in Figure 2.c in which the user can also access the touchscreen of the smartphone/tablet.

We provide in Figure 3 the possible interactions for each implementation described above. These interactions are :

- Exploration of content, in which users simply reveal the static virtual content by moving the device through it

- Triggering events, in which users can activate dynamic content (e.g. animations, videos, sounds ...)

- Thematic selection, in which users can select between a set of virtual content placed at the same physical position, for example to allow for multiple information regarding the same physical object. An example of that is used in our preliminary study in Section 4.1 where visitors can access information related to either the habitat or food of naturalised birds.

- Content manipulation, in which users can interact with the content in a more advanced way than only triggering and filtering, e.g. scrolling through a map, manipulating data diagrams.

- Content creation refers to the possibility of adding content, for example leaving messages or annotation on the exhibited objects, through the mobile touchscreen for example.

- Content capture refers to the possibility of capturing the revealed content, either manually by tracing it on the transparent panel implementation, or by taking a picture and screenshot with the mobile implementation. 


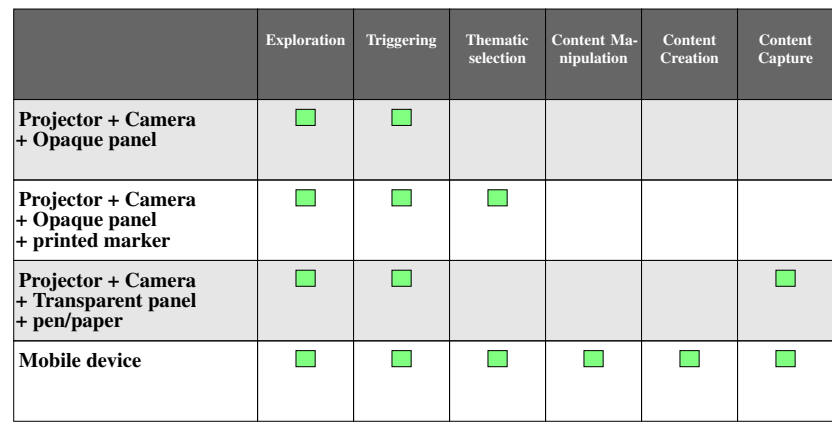

Figure 3: Possible interactions with virtual content depending on the implementation of RVDs

\subsubsection{Cabinet viewing side}

The last design dimension in RVD which influences interaction possibilities is the side of the glass cabinet through which objects are viewed. Exhibited artefacts will be either perceived mostly through a vertical side, such as seen in Figure 2.a or through the top side, from above as seen in Figure 2.b. In the case of vertical sides, artefacts can be placed at any distance from the surface, i.e. on the reflected axis, which may lead to more time finding the content as this reflected axis seems more difficult to control, as explained in more details in the following experiments. With the horizontal top side, objects are usually closer to the panel, requiring less adjustments on the reflected axis and making the exploration of content potentially faster. Horizontal sides may however make the content less visible since visitors can be placed all around the case, not necessarily facing the panels used to reveal the content, and not all in front of it as with vertical sides.

\section{Facilitating 3D exploration With a Revealable VOLUME DISPLAY}

In this section we describe two experiments that investigate the appropriation of RVDs by users and how to facilitate exploration with them. Both of them were conducted before the COVID-19 pandemic, in the summer and fall of 2018. These experiments use the projector + camera + opaque panel implementation. However the other RVDs implementations suffer from the same perceptual issues and should therefore benefit from the proposed techniques.

\subsection{Preliminary study}

The purpose of this first study was to investigate information exploration by individuals and to evaluate the appropriation of our prototype in an ecological situation. Due to time constraints of conducting the study in a museum setting, we focused on gathering qualitative data.

The prototype was trialed in a regional natural history museum. It was deployed in a showcase containing naturalised birds (see Figures 4.a and 4.b). For each bird we provided their name, diet, living environment and number of laid eggs. 30 participants (15 groups of 2 participants each) were recruited for the experiment in two sessions in the museum. Participants included 15 males and 15 females with a mean age of $27.3(\mathrm{sd}=12.37, \min =8, \max =52)$. None of them had previously participated in similar studies or used our system but some had experience with augmented reality (AR experience rated between $1-5$ with mean $=2.3, \mathrm{sd}=1.34$ ). They all participated voluntarily and signed an informed consent form. The duration of the experiment was between 10 and 15 minutes.

The experiment involved pairs of users (to allow for a collaborative task) each facing half of the showcase (see figures 4.a and 4.b). Before each task, subjects were allowed to spend enough time to get

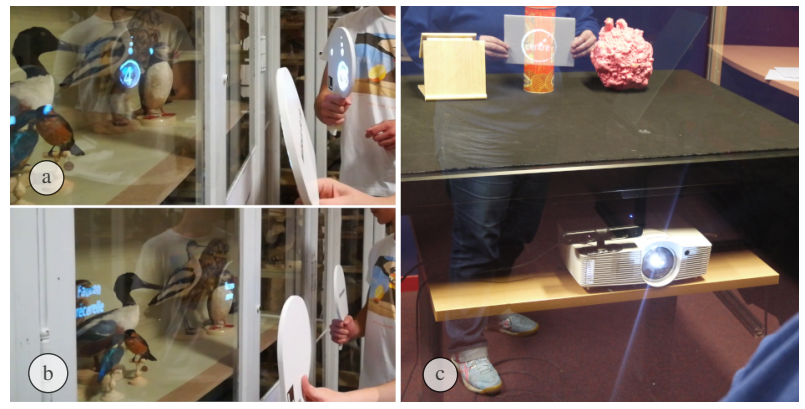

Figure 4: Setup for the two experiments: (a) Experiment 1 : Introduction phase where participants reveal the names of the birds, (b) Experiment 1 : Exploration phase in which the average number of eggs laid by each bird is attached to it with a 3D guide. (c) Experiment 2

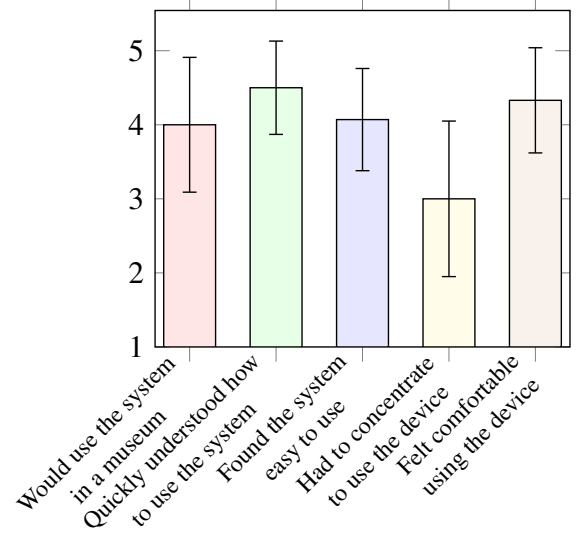

Figure 5: Questionnaires results from the first experiment

familiar with the device. The experiment was divided into two steps

- In the first step, participants were introduced to the prototype and told how to interact with it. They were given time to experiment until they felt accustomed to it.

- The second step was dedicated to the trial of navigation guides to help users find relevant information around the exhibits. The users were informed that starting from the head of the animals, they would find a guide to follow to find information. They were asked to find information about the number of eggs laid by each animal and to identify the one having the more (resp. less) eggs. Three kinds of guides were evaluated. This aspect of our system will be described in more details in the second experiment, as no significant results were obtained. Each pair of users had the same guides and we alternated between groups.

The trials were recorded for later analysis of the users' interactions with the device and comments. After each step, each user passed a questionnaire with 5 levels Likert items to assess usability and acceptance of the system. Results from the questionnaires in the first and the second step are shown in Figure 5.

Although the feedback from participants of this first experiment was very positive as shown by the results of our questionnaires, the observation of participants in an ecological situation revealed that exploration with the system remains difficult, see Figure5. In particular they seemed to have trouble finding the virtual content 
around the exhibited objects. We believe this is due to the reflected interaction, which reverses displacements along the z-axis (towards or away from the glass panel), and to the limited visual depth cues. In fact, visual cues for depth perception such as parallax, occlusions, shading and shadows are lost with the RVD. This causes difficulties of perception and navigation for users and may hinder their experience in the museum. We therefore decided to investigate how individual exploration can be facilitated with a second, more controlled experiment.

\subsection{Controlled experiment}

This controlled experiment seeks to answer the following questions:

- Does having the contour of physical objects virtually highlighted improve the positioning around the physical objects ?

- Do animated guides make it easier to find to which object or part of an object virtual content is attached, compared to static links (3D lines) ?

- Does highlighting depth contours for the virtual content help users discover this content?

20 participants (16 males, 4 females) were recruited for the experiment (aged mean=31.10 SD=11.17 $\min =18 \max =61$ ). None of them had previously participated in similar studies. They participated voluntarily after signing and informed consent form. Results from a 21 st participant were removed because they had trouble adjusting to the system, resulting in very low performance compared to the other participants. The experiment was performed in a quiet, illuminated laboratory room. Before each task subjects were allowed to spend enough time to get familiar with the device.

The experiment lasted around 30 minutes. For each task participants were holding an A5 piece of white foam board with their two hands to drive the projection. The glass cabinet contained three physical objects. We used an Optoma 1080E projector with a 1920x1080 resolution at 60fps, combined with an Asus Xtion providing a $320 \times 240$ depth image at $60 \mathrm{fps}$. The experimental setup is shown in Figure 4.c.

The experiment followed a within-subject design. It was divided into three tasks : CONTOURS, GUIDED, CONTENT. We added a fourth task named CONTENT2, based on initial results, that the participants performed in a second session. Each task had two conditions. Between each condition participants filled a subjective questionnaire with 5 levels Likert items to gather their impressions about the task. At the end of each task, they also selected their preferred condition. CONTENT 2 was designed to refine the results from CONTENT, as post task interviews revealed an effect of the two conditions that was not visible in the data. It was conducted between 2 and 7 days after the first experiment on only 12 of the participants of CONTENT (11 males, 1 female, aged of mean=30.58, $\mathrm{SD}=11.46, \min =18, \max =61$ ).

\subsubsection{Contours task}

The goal of this task was to evaluate whether highlighting the physical objects with their virtual contour helps users in their exploration, i.e. if they better perceive where the reflection of the panel they are holding is, relatively to the physical objects. At the beginning of each trial, participants were asked to reveal a virtual sign indicating around which object a virtual stationary red sphere would be placed and where around the object it would be (e.g. "above to the left"). As soon as the sign disappeared they had to find the sphere as fast as possible and remain on it until it disappeared (after 2 seconds). The sphere was placed near one of the 3 objects and at one of 4 positions around the object (right, left-behind, left-top-behind, rightbehind) (at $\sim 50 \mathrm{~mm}$ from the contour of the object). Each participant therefore performed 12 trials $(3 * 4)$ per condition and the trials were randomised. Two conditions were evaluated: no contours for the

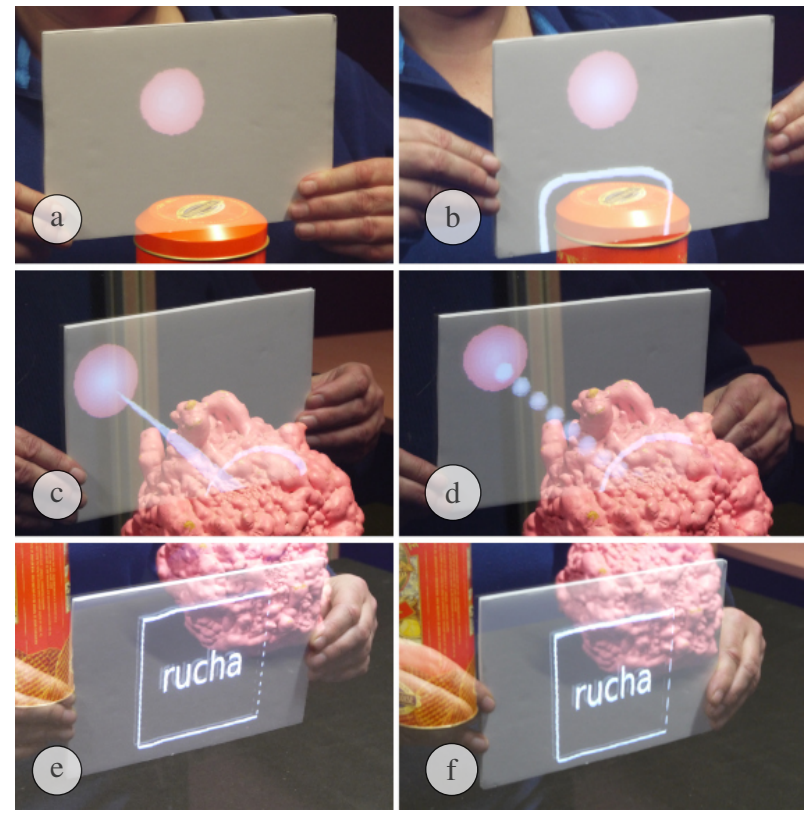

Figure 6: Experiment 2 : Conditions for the three tasks. Contours Task : CONTOUR_NO (a) and CONTOUR_YES (b). Guides Task : GUIDE_STATIC (c) and GUIDE_DYNAMIC (d). Content Task : $F R A M E \_F L A T$ (e) and FRAME_DEPTH (f)

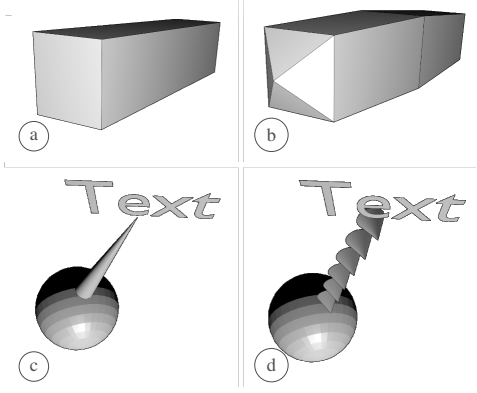

Figure 7: Techniques used in the Content and Guides Tasks : (a) FRAME_FLAT (b) FRAME_DEPTH (c) GUIDE_STATIC (d) GUIDE_DYNAMIC

physical objects (CONTOURS_NO) and presence of the contours (CONTOURS_YES). These two conditions are depicted in Figure 6.a and Figure6.b.

\subsubsection{Guides task}

We seek to guide users from a specific part of a physical object to a piece of information, in order to facilitate annotations of the physical objects with "floating" virtual content. For that, we trialed animated guides (GUIDE_DYNAMIC) that give a clue of the direction to follow with a series of 3D cones moving towards the target and static guides (GUIDE_STATIC) that link the object and the information with a single 3D cone. These two guides are depicted in Figures 7.c 7.d, and the result when revealing both the name and red sphere with both guides is shown in Figures 6.c and 6.d.

We chose not to test any guide which would require the system to know the position of the user, e.g. 3D arrows in perspective, in order to maintain the public and shared aspects of RVDs.

Each trial started with a virtual sign indicating the object around 
which a virtual red sphere was going to be. As soon as the virtual sign disappeared, they had to move to the designated object, reveal its name (which was placed in the centre of the object) and then find the stationary sphere as quickly as possible. They then had to remain on the sphere until it disappeared (after 2 seconds) and then move back to the sign for the next trial. The sphere was placed around one of the three objects in two possible directions (right+above, left+above) and two planes (same depth as the object / closer to the user). This resulted in 12 trials $(3 * 4)$ per condition for each subject and the order of trial was randomised.

\subsubsection{Content task}

With this task, we investigate how to improve depth perception during the exploration of virtual content especially when the content does not change much along the depth axis (few images, text, compared to 3D text). In the task, participants were asked to first remain on a virtual sign with instructions. As soon as this sign disappeared, they had to find a word placed in front of or behind the virtual sign as quickly as possible and say the word out loud (without necessarily remaining on the word). After this, the word disappeared and they moved back to the virtual sign for the next trial. Two conditions were evaluated. In FRAME_FLAT the word was surrounded by a simple box, shown in Figure 7.a. In FRAME_DEPTH, the shape of the frame surrounding the word quickly increased from $0 \%$ to $95 \%$ of the box size at both extremities, and then increased slowly along the depth to reach $100 \%$ in the middle of the box. This shape is depicted in Figure 7.b. The FRAME_DEPTH shape was designed so as to show almost no visual difference with the FRAME_FLAT shape, in order to preserve the maximum space for virtual content placed inside. This absence of difference is visible in Figures 6.e and 6.f. Both shapes were of $100 \mathrm{~mm}$ in width and $100 \mathrm{~mm}$ in height. Between trials, we varied direction of the frame (i.e. behind / before of panel), the distance of the frame to the panel (100mm and $150 \mathrm{~mm})$ and the depth of the frame $(60 \mathrm{~mm}$ and $150 \mathrm{~mm})$ together with the depth of the word placed at the centre of the frame (occupying a third of the depth of the frame, so respectively $20 \mathrm{~mm}$ and $50 \mathrm{~mm}$ ). Each participant therefore performed 8 trials ( 2 directions $* 2$ distances $* 2$ depths) per condition and the trials were randomised. We measured the number of errors (finding and loosing the word), and the time taken to find the word once inside the frame.

\subsubsection{Content2 task}

The initial Content task was modified to require more precision from the participants. To that extent, the thickness of the word that participants had to find was reduced from $50 \mathrm{~mm}$ and $20 \mathrm{~mm}$ to $20 \mathrm{~mm}$ and $10 \mathrm{~mm}$. We also varied the depth of the frame separately between $60 \mathrm{~mm}$ and $150 \mathrm{~mm}$. Each participant therefore performed 16 trials ( 2 directions $\mathrm{x} 2$ frame depth $\mathrm{x} 2$ frame distance $\mathrm{x} 2$ word thickness) per condition and the trials were randomised. In addition, they were asked to find the word as fast as possible and more importantly to remain on it until it disappeared (after 2 seconds). The same conditions were evaluated as in the Content task (i.e. FRAME_FLAT and FRAME_DEPTH).

\subsection{Results}

Here we give all statistically significant differences observed between conditions. They are also depicted in Figure 8. The implications of these results are discussed in Section Guidelines. We provide both frequentist and bayesian analyses, these were performed using JASP $0.13 .1^{1}$. We used a Student t-test if the normality was verified with a Shapiro-Wilk test, otherwise we used a Wilcoxon signed rank non-parametric test. Bayesian statistics were performed to provide a finer grained analysis of the effects (e.g. weak, moderate, strong ...), using the interpretation of Bayes factors from JASP, in turn adapted from Jeffrey's scheme [24].

${ }^{1}$ https://jasp-stats.org
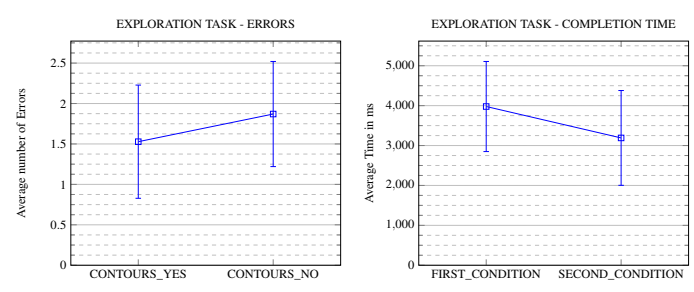

GUIDES TASK - REENTRIES
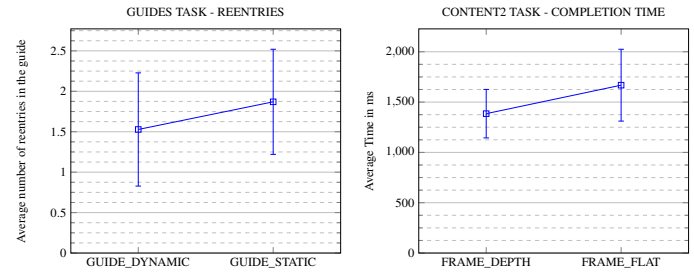

Figure 8: Plots of the statistically significant results in the Contours, Guides and Content tasks.

\subsubsection{Contours task}

Before analysing the results, we removed the outliers which were trials that lasted for more than 20 s, correlated with the participant forgetting the instruction, i.e. around which object the target was. Adding a virtual contour around the objects (CONTOUR_YES) resulted in less errors (mean $1.528, s d=0.768$ ) that without them $\left(C O N T O U R \_N O\right.$, mean $\left.=1.870, s d=0.65\right)$. A Student's t-test $(t(19)=-2.316, p=0.032)$ confirms that the difference is statistically significant. The corresponding Bayes factor $\left(B F_{10}=1.985\right)$ shows a weak evidence for a difference. Regarding completion time, a Bayesian t-test reveals a moderate evidence for an absence of difference between the conditions $\left(B F_{10}=0.232\right)$. There is also a statistically significant difference $(\mathrm{t}(19)=3.214, \mathrm{p}=0.005)$ when comparing task completion time (in milliseconds) between the first condition participants had and the second one. This duration reduces (mean = $3978.776 \mathrm{~ms}, \mathrm{sd}=1129.721$ ) between the first condition (no matter which one it was) and the second (mean $=3191.554$ mssd $=$ 1188.005). The corresponding Bayes factor $\left(B F_{10}=9.908\right)$ also shows a strong evidence for a difference. We also compared the results between participants who had CONTOUR_YES first $(\mathrm{n}=11)$ and participants with $C O N T O U R \_N O$ first $(\mathrm{n}=9)$, which leads to the following observations :

- In terms of errors, there is a statistically significant difference depending on whether the users started with CONTOURS_YES or CONTOURS_NO $(t(18)=-2.618, p=0.017)$. The errors increased when they passed from CONTOURS_YES to CONTOURS_NO (mean $=0.114, s d=0.728)$. The errors decreased in the reverse condition ( mean $=-0.620, s d=0.460$ ). A Bayesian independent t-test confirms that there is moderate evidence for a difference $\left(B F_{10}=3.485\right)$.

- There is also a statistically significant difference in terms of reentries in objects $(t(18)=-2.311, p=0.033)$ but with only a weak evidence in Bayesian analysis $\left(B F_{10}=\right.$ 2.247). The number of reentries increased when passing from CONTOURS_YES to CONTOURS_NO (mean = $0.202, s d=1.909)$ and decreased in the reverse order $($ mean $=$ $-1.415, s d=0.947)$.

\subsubsection{Guides task}

The results do not show statistically significant differences in completion time or errors between the two kinds of guides. Bayesian analysis shows a moderate evidence for an absence of difference in errors $\left(B F_{10}=0.242\right)$ and completion time $\left(B F_{10}=0.274\right)$. However we 
can note a statistically significant difference $(t(19)=2.783, p=$ 0.012 ) in reentries in guides (i.e. leaving the guide and entering it again) with less reentries when using GUIDE_DYNAMIC (mean $=4.4, s d=1.7)$ compared to GUIDE_STATIC (mean = $5.612, s d=1.488)$. This is confirmed by a moderate evidence for a difference $\left(B F_{10}=4.447\right)$. Participants finally expressed a preference towards dynamic guides (13 against 7).

\subsubsection{Content 1 and 2 tasks}

Data from Content 1 does not show any statistically significant difference either in terms of completion time or errors. However a majority of participants reported differences that could indicate a change in depth perception, although they could not tell what the difference between the conditions was. Among the 20 participants: 8 perceived no difference between the two conditions; 3 felt that the FRAME_DEPTH condition was easier; the remaining 9 perceived a difference related to distance perception : 5 users felt that the word was closer in FRAME_DEPTH condition, 3 felt that the word were thinner in FRAME_DEPTH and 1 that they could reach the word faster in FRAME_DEPTH.

The results from the second iteration of the task Content 2 , which required more precision (due to thinner words), show a statistically significant difference in word finding time $(\mathrm{t}(11)=-2.840, \mathrm{p}=0.016)$ with lower duration for FRAME_DEPTH (mean=1385.208 $\mathrm{ms}, \mathrm{sd}=241.669)$ than for FRAME_FLAT (mean $=1668.667 \mathrm{~ms}$, $\mathrm{sd}=357.446)$. This is confirmed by a Bayesian t-test showing a moderate evidence for a difference $\left(B F_{10}=4.003\right)$.

\section{Discussion ANd GUIDELINES}

Even though we found a number of statistically significant differences between the techniques in each task, these remain quite small : less than 1 second for the completion time and only one or two additional errors. However, these were obtained on very short and guided tasks, i.e. participants knew what they were supposed to find and they were only looking for one information at a time. We believe that these small differences might accumulate and become more problematic in ecological conditions where visitors do not always know precisely what they are looking for, where there are more objects and virtual content and where visitors might get frustrated and stop using the system if they feel like they are not able to achieve the task fast enough. In the following, we propose guidelines based on our results for designing interaction with RVDs to optimise the user experience. Although we only tested the projector + camera + opaque panel implementation, we believe they can ve applied to all implementations of RVDs.

\subsection{Progressive complexity}

Results of the Contours task indicate that there is a short learning curve for RVDs. In fact participants became a lot more confident and accurate over time. We therefore believe that in order to facilitate the use of RVDs, a trajectory could be defined that would start with a cabinet featuring only a few simple virtual annotations on a single object and increase complexity and diversity of the content progressively as spectators progress into the exhibitions. Another possibility is to use the thematic selection that we presented in Section 3.3 to filter out some of the content for novices who would use a panel with a special AR tag.

\subsection{Dynamic 3D Guides}

In order to attach virtual content such as annotations around exhibited objects, a method for guiding visitors to the content needs to be added. Instead of relying on text indications ("move upwards") or arrows which require the system to know which content visitors are looking for, we proposed two types of virtual guides simply created by placing $3 \mathrm{D}$ shapes in the physical space between the physical object and the annotation. Even though there were no differences in performance times, results of our study show that participants mostly preferred the dynamic guides and that they lost them less often than when following the static guides. It therefore seems that dynamic guides with an animation showing the direction of the virtual content are to be picked in priority. In addition, dynamic guides led to a strategy of slowly following the guides (without loosing them), while static guides led to a strategy where users would scan the volume with fast and large movements. We believe that slower movements should be encouraged as they will help visitors explore more complex scenes of virtual content, where large movements would make them miss some of the content, especially with tracking and projection latency issues. Our recommendation is therefore to rely mostly on dynamic guides.

\subsection{Highlighting contours of physical objects}

When interacting with an RVD, depth perception cues such as occlusions, shading and shadows are lost. It is therefore difficult for visitors to precisely perceive where the reflection of the surface they are holding is, relative to the exhibited objects. Our study suggests that less errors were made in finding virtual content of a physical object when the contours of that object were highlighted, and the same contours led to a stronger reduction of errors over time. This might indicate that participants had a better perception of position within the display case / around the object when given those contours. They might therefore serve as an anchor in the exploration around physical objects. The presence of contours also led participants to use slower movements in order to keep the contour visible at all times. We believe this induced behaviour would also improve the overall experience of RVDs, since visitors will move with more precision and stability through the virtual content when exploring. Implementing this contours technique however implies that all exhibited objects are scanned and the obtained 3D meshes are aligned with them.

\subsection{Highlighting depth inside virtual content}

Another effect of the impaired depth perception in RVDs is that it can be difficult for visitors to know their position along the z-axis when revealing virtual content. Findings from our study seem to indicate that amplifying this displacement with changes in the content or of a frame surrounding the content leads to faster selection of content along the z-axis, even if the changes are not consciously perceived by the users. Although the time difference remains small (300ms) in our task with a single word that had to be revealed, it could lead to much larger differences when accumulated during the exploration of more complex content. We recommend using depth highlighting frames, i.e. shapes that change contour when one moves forward and backward. A very small change such as the one used in our study seems to already restore a sense of depth. We believe that stronger changes with for example spherical frames, depth layered images or 3D textures would also help visitors to better perceive their displacement inside the museum cabinet.

\section{CONCLUSION}

In this paper, we presented the concept of Revealable Volume Displays, mixed-reality displays for 3D exploration in public exhibitions. We described how they can be implemented and what interaction possibilities they afford. Following an in-situ experiment on their appropriation by museum visitors, we conducted a controlled experiment to investigate techniques for facilitating their use. Our results suggest that virtual contours, dynamic guides and amplified depth movements can help reduce exploration time and errors and lead users to more stable and precise content exploration. The projector + camera implementation with added contours and guides has been used on several occasions since the experiment, and is being integrated in upcoming exhibitions. Among the main perspectives, the other RVDs implementations presented will be investigated further to see if additional guiding techniques are required. 


\section{REFERENCES}

[1] L. T. E.-J. Anders Ynnerman, Jonas Löwgren. Exploranation: A new science communication paradigm. IEEE Computer Graphics and Applications, 72, 08 2018. doi: 10.1109/MCG.2018.032421649

[2] M. K. Bekele, R. Pierdicca, E. Frontoni, E. S. Malinverni, and J. Gain. A survey of augmented, virtual, and mixed reality for cultural heritage. Journal on Computing and Cultural Heritage (JOCCH), 11(2):7, 2018.

[3] S. Benford, G. Giannachi, B. Koleva, and T. Rodden. From interaction to trajectories: Designing coherent journeys through user experiences. In Proceedings of the SIGCHI Conference on Human Factors in Computing Systems, CHI '09, pp. 709-718. ACM, New York, NY, USA, 2009. doi: $10.1145 / 1518701.1518812$

[4] F. Berthaut, C. Arslan, and L. Grisoni. Revgest: Augmenting gestural musical instruments with revealed virtual objects. In International Conference on New Interfaces for Musical Expression, 2017.

[5] L. Besançon, A. Ynnerman, D. F. Keefe, L. Yu, and T. Isenberg. The state of the art of spatial interfaces for $3 \mathrm{~d}$ visualization. In Computer Graphics Forum. Wiley Online Library, 2020.

[6] O. Bimber and R. Raskar. Spatial Augmented Reality Merging Real and Virtual Worlds, vol. 176. 2005. doi: 10.1503/cmaj.070353

[7] D. L. Boisvert and B. J. Slez. The relationship between visitor characteristics and learning-associated behaviors in a science museum discovery space. Science Education, 78(2):137-148, 1995. doi: 10.1002/sce. 3730780203

[8] S. Burigat, L. Chittaro, and S. Gabrielli. Visualizing locations of offscreen objects on mobile devices. p. 239, 2006. doi: 10.1145/1152215. 1152266

[9] M. B. Carmo, A. P. Afonso, A. Ferreira, A. P. Cláudio, and G. Silva PoI awareness, relevance and aggregation for augmented reality. Proceedings of the International Conference on Information Visualisation, 2016-Augus:300-305, 2016. doi: 10.1109/IV.2016.47

[10] P. Cash and C. Snider. Investigating design: A comparison of manifest and latent approaches. Design Studies, 35(5):441-472, 2014. doi: 10. 1016/j.destud.2014.02.005

[11] G. Casiez, N. Roussel, and D. Vogel. $1 €$ filter: a simple speed-based low-pass filter for noisy input in interactive systems. In Proceedings of the SIGCHI Conference on Human Factors in Computing Systems, pp. 2527-2530. ACM, 2012.

[12] A. Cassinelli and M. Ishikawa. Volume slicing display. In $A C M$ SIGGRAPH ASIA 2009 Art Gallery; Emerging Technologies, pp. 8888, 2009. doi: 10.1145/1665137.1665207

[13] K.-E. Chang, C.-T. Chang, H.-T. Hou, Y.-T. Sung, H.-L. Chao, and C.M. Lee. Development and behavioral pattern analysis of a mobile guide system with augmented reality for painting appreciation instruction in an art museum. Computers \& Education, 71:185-197, feb 2014. doi: 10.1016/j.compedu.2013.09.022

[14] C. J. Chen and W. M. F. Wan Ismail. Guiding exploration through three-dimensional virtual environments: A cognitive load reduction approach. Journal of Interactive Learning Research, 19(4):579-596, 2008.

[15] P. Galatis, D. Gavalas, V. Kasapakis, G. Pantziou, and C. Zaroliagis Mobile augmented reality guides in cultural heritage. MobiCASE 2016 - 8th EAI International Conference on Mobile Computing, Applications and Services, (January), 2016. doi: 10.4108/eai.30-11-2016.2266954

[16] T. A. Galyean. Guided navigation of virtual environments. Proceedings of the Symposium on Interactive 3D Graphics, (January 1995):103-104, 1995. doi: 10.1145/199404.199421

[17] C. Heath. Interaction and interactives: collaboration and participation with computer-based exhibits. Public Understanding of Science, 14(1):91-101, jan 2005. doi: 10.1177/0963662505047343

[18] S. Hsi. A study of user experiences mediated by nomadic web content in a museum. Journal of Computer Assisted Learning, 19(3):308-319, sep 2003. doi: 10.1046/j.0266-4909.2003.jca_023.x

[19] M.-B. Ibáñez and C. Delgado-Kloos. Augmented reality for stem learning: A systematic review. Computers \& Education, 2018.

[20] H. Kajita, N. Koizumi, and T. Naemura. Skyanchor: Optical design for anchoring mid-air images onto physical objects. In Proceedings of the 29th Annual Symposium on User Interface Software and Technology - UIST '16, p. 415-423. ACM Press, 2016. doi: 10.1145/2984511.
2984589

[21] A. Karnik, W. Mayol-Cuevas, and S. Subramanian. Mustard: a multi user see through ar display. In Proceedings of the SIGCHI Conference on Human Factors in Computing Systems, pp. 2541-2550. ACM, 2012.

[22] J. Keil, M. Zöllner, M. Becker, F. Wientapper, T. Engelke, and H. Wuest. The House of Olbrich An Augmented Reality tour through architectural history. 2011 IEEE International Symposium on Mixed and Augmented Reality - Arts, Media, and Humanities, ISMAR-AMH 2011, pp. 15-18, 2011. doi: 10.1109/ISMAR-AMH.2011.6093651

[23] F. Liarokapis, M. White, and P. Lister. Augmented reality interface toolkit. Proceedings of the International Conference on Information Visualization, 8(June 2014):761-767, 2004. doi: 10.1109/iv.2004. 1320227

[24] A. Ly, A. J. Verhagen, and E.-J. Wagenmakers. Harold jeffreys's default bayes factor hypothesis tests: Explanation, extension, and application in psychology. Journal of Mathematical Psychology, 72, 08 2015. doi: 10.1016/j.jmp.2015.06.004

[25] D. Martinez Plasencia, F. Berthaut, A. Karnik, and S. Subramanian. Through the combining glass. In Proceedings of ACM UIST, pp. 341350, 2014. doi: 10.1145/2642918.2647351

[26] E. Mayr and D. Wessel. Potentials and Challenges of Mobile Media in Museums. In Proceedings Interactive Mobile and Computer Aided Learning (IMCL), vol. 1, 2007.

[27] B. Ridel, L. Mignard-Debise, X. Granier, and P. Reuter. Egosar: Towards a personalized spatial augmented reality experience in multi-user environments. In Mixed and Augmented Reality (ISMAR-Adjunct), 2016 IEEE International Symposium on, pp. 64-69. IEEE, 2016.

[28] B. Ridel, P. Reuter, J. Laviole, N. Mellado, N. Couture, and X. Granier. The revealing flashlight: Interactive spatial augmented reality for detail exploration of cultural heritage artifacts. Journal on Computing and Cultural Heritage (JOCCH), 7(2):6, 2014.

[29] F. Romero Ramirez, R. Muñoz-Salinas, and R. Medina-Carnicer. Speeded up detection of squared fiducial markers. Image and Vision Computing, 76, 06 2018. doi: 10.1016/j.imavis.2018.05.004

[30] T. Schinke, N. Henze, and S. Boll. Visualization of off-screen objects in mobile augmented reality. ACM International Conference Proceeding Series, (January):313-316, 2010. doi: 10.1145/1851600.1851655

[31] S. Schmidt, F. Steinicke, A. Irlitti, and B. H. Thomas. Floor-Projected Guidance Cues for Collaborative Exploration of Spatial Augmented Reality Setups. ISS 2018 - Proceedings of the 2018 ACM International Conference on Interactive Surfaces and Spaces, pp. 279-289, 2018. doi: $10.1145 / 3279778.3279806$

[32] R. Sodhi, H. Benko, and A. Wilson. Lightguide: Projected visualizations for hand movement guidance. In Proceedings of ACM CHI, pp. 179-188, 2012. doi: 10.1145/2207676.2207702

[33] C. Sun, X. Chang, M. Cai, and J. Liu. An Improved Design of 3D Swept-Volume Volumetric Display. Journal of Computers, 9(1):235242, 2014. doi: 10.4304 jcp.9.1.235-242

[34] D. vom Lehn, C. Heath, and J. Hindmarsh. Video-based Field Studies in Museums and Galleries. Visitor Studies Today, V(III):15-23, 2002.

[35] C. Wu, M. Zollhöfer, M. Nießner, M. Stamminger, S. Izadi, and C. Theobalt. Real-time shading-based refinement for consumer depth cameras. ACM Transactions on Graphics (ToG), 33(6):200, 2014.

[36] I. Yahya. Museum Learning, The Museum Visitor, The Museum Visit An. PQDT - UK \& Ireland, (June 1997):338, 1997.

[37] E. Yasui. Collaborative idea construction: Repetition of gestures and talk in joint brainstorming. Journal of Pragmatics, 46(1):157-172, 2013. doi: 10.1016/j.pragma.2012.10.002 